\title{
Study on Effect of MRI Scanner on Brain Tumour Detection
}

\author{
Vandana Shah \\ Research Scholar \\ Electronics \& Communication \\ MIET, Gondia
}

\author{
Vijay Chourasia \\ Assistant Professor \\ Electronics \& Communication \\ MPIET, Gondia, Nagpur, India
}

\author{
R.V.Skhirsagar \\ Professor \\ Electronics \& Communication \\ PCE, Hingna, Nagpur, India
}

\begin{abstract}
Now a day the technology took the paradigm shift in the area of medical field. It is now easy to diagnose the biological problems through different medical devices like CT scan, MRI, and X-rays etc. Tumor is an uncontrolled development of tissues in any piece of the body. Mind tumor is naturally genuine and life-debilitating due to its character in the constrained space of the intracranial hole. By and large, CT output or MRI that is coordinated into intracranial depression delivers a complete picture of cerebrum and this picture is outwardly analyzed by the doctor for identification and determination of mind tumor. In India 1.5 to 3 Tesla scanners are mostly used. But both have different Magnetic field effect. Because of the the different strength of Magnetic field there is a possibility of noise present in the image. This can create problem in detection of the accurate determination of location and size of tumor. Hiding patient information for further analysis is also very crucial for the confidentiality. security algorithm takes care for the hidden system and analysis of the patient. The survey identifies the efficient algorithm for finding the accurate tumor and also identifies its normality.
\end{abstract}

\section{General Terms}

Image Denoising, Image segmentation, Image classification System Security, Algorithms.

\section{Keywords}

MRI image, Tesla, Discrete Wavelet Transform, Neural Network.

\section{INTRODUCTION}

Exposure to radiation either as a result of a nuclear accident or during treatment of diseases may increase the risk of brain tumours in people under the age of 30 , says an Indian-origin specialist. Researchers analysed records of 35 patients, who were diagnosed with a form of brain tumour called meningiomas before the age of $30^{[24]}$. Between 10 and $20 \%$ of brain tumors in children are brain stem gliomas. This tumor most often affects children between 5 and 10 years old, but can also be found in adults generally between 30 and 40 years old. Most of these tumors are astrocytomas which vary from localized grade I tumors (mostly in children) to infiltrating grade II or III tumors. Each year more than 66,000 Americans are diagnosed with a primary brain tumor and more than twice that number are diagnosed with a metastatic tumor ${ }^{[25]}$.

The information stated above is as per the survey of WHO (World Health Organization) and ABTA (American Brain Tumour Association) also, lamentably, it is extremely basic for mind tumor patients to experience side effects connected with their tumor(s) and/or treatment(s). Individuals with cerebrum tumors frequently experience the ill effects of: Headaches, Seizures, Sensory (touch) and engine (development control) misfortune, Deep venous thrombosis (DVT, or blood clump), Hearing misfortune, Vision misfortune, Fatigue, Depression, Behavioral and psychological (considering) changes, Endocrine brokenness (hormone/organ changes). These manifestations may be connected with the sort, size, and/or area of the tumor, and the medicines used to oversee it. Surgery, radiation, chemotherapy, and different medicines all can possibly create new indications as they work to lessen the effect of the tumor. Below figure 1 shows the real data of different patient's Brain images.

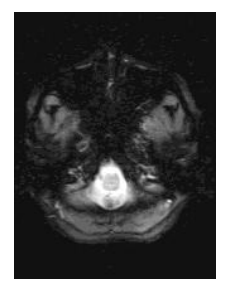

(a)

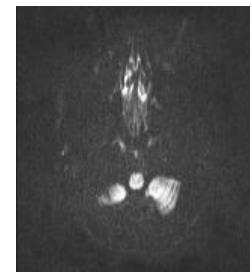

(d)

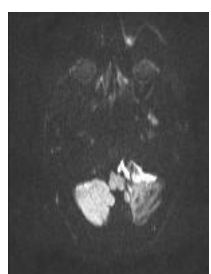

(b)

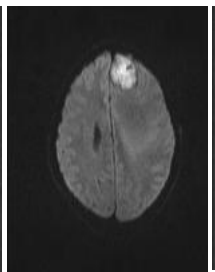

(e)

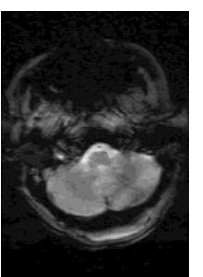

(g)

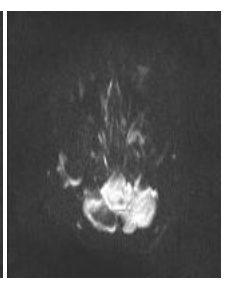

(c)

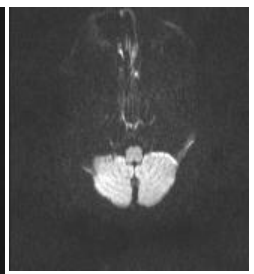

(f)
Fig 1: Different brain images real data of patients. ${ }^{[26]}$

Above figure 1 shows the different images of brain tumor which are classified as: (a). Brainstem TB tumor (b). Epidermoid cyst (c). Posterior fossa pilocyastic astrocytoma (d). Gbm post radiotherapy lesion (e). Glioma mets meningioma. (f). Glioblastoma. (g). Meningioma. These MRI images are in JPEG formats which are converted from DICOM images. 

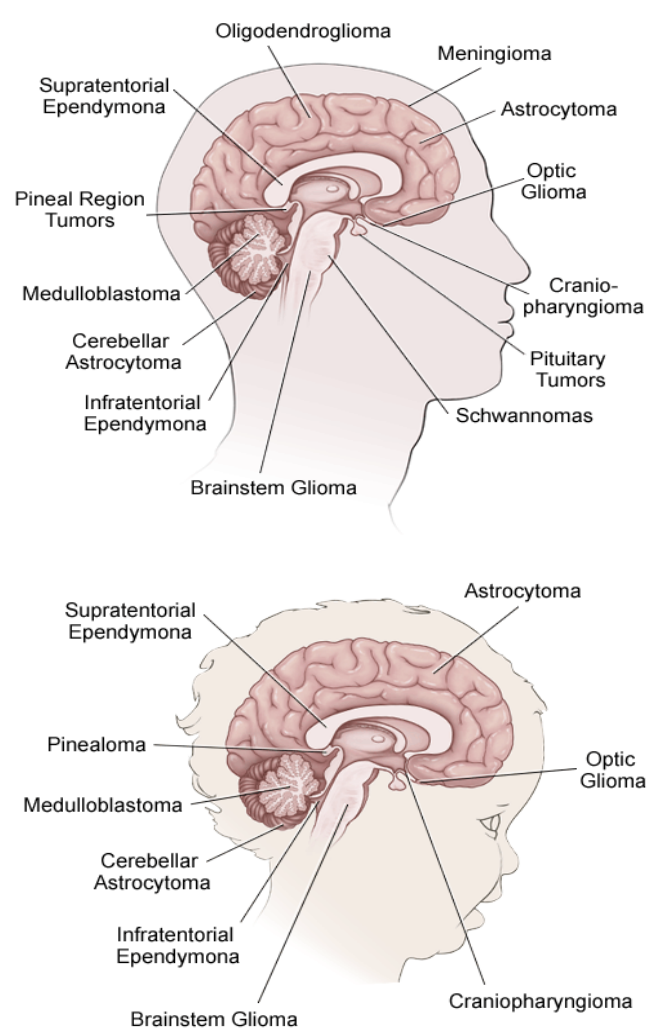

\section{Fig 2: Locations of different types of brain tumors in adult and a child. ${ }^{[27][28]}$}

Figure 2 shows the Tumor locations in adult and a child. There are 13 places where tumors can be located in adult and 9 places where the tumors can be located in the small children of the approximate age of 10 or below 10 .

\section{RATIONAL FOR SELECTING THE PROBLEM}

As per the above mentioned survey we can state that number of patient losing their life due to brain tumour is drastically increases. This scenario is leading to very dangerous and it gives lot of challenges medical and engineering field. To avoid the above stated problem, this research work is proposed for removing the noise from MRI images using the best technique found after survey. And also the image segmentation technique is proposed for detection of brain tumour as well as other parameters. This method allows the extraction of tumour tissue with accuracy and also reduces the time for analysis. Image Denoising problem is very important in many application and it is preprocessing step for image processing where zero mean and white additive Gaussian noise is to be removed from a given noisy image.

Existing techniques for Denoising of image is having drawbacks of losing high frequency component containing fine details. The proposed algorithm may lead to effective noise removal technique and also reduce computational complexity with better noise reduction performance. After denoising certain image segmentation algorithms for the MRI image of the brain are studied. While doing segmentation the major problem is in few algorithm is to define few parameters by the user and even it is very much unpredictable to justify the accuracy of the implemented algorithm, moreover the computational time required is the major issue for the efficient technique. As most of these approaches are parametric and require the approximate initial number of clusters to proceed further the proposed algorithm is identified. Image denoising and Image segmentation is an important and challenging problem in image analysis and diagnosis as per the survey.

The objectives of the proposed work are: Noise reduction, as it is having vast application in Magnetic Resonance imaging, Astronomical imaging, satellite imaging where visual quality of image plays an important role in terms of accuracy. Study different architectures for clustering for image segmentation and analyse the performance in terms of Time Computation and Accuracy. For certain applications the image Denoising could be used to remove noise from the original image. Further research will also highlight the different technique in detail for image denoising like wavelet domain and spatial frequency domain etc. Secondly the image is pre-processed by a standard clustering based segmentation which preserves the desirable discontinuities present in the image and guarantees over segmentation in the Output.

The upsides of the proposed division are it has low computational intricacy and is consequently exceptionally achievable for continuous picture division preparing. It preprocesses a picture by utilizing the proposed calculation to shape fragmented districts that save the alluring irregularity qualities of the picture ${ }^{[2]}$. The proposed strategy permits a low-dimensional picture grouping with huge lessening of the unpredictability contrasted with traditional diagram parceling routines that are straightforwardly connected to the picture pixels[2]. Existing picture division calculations can be for the most part grouped into three noteworthy classes, i.e., highlight space-based bunching, spatial division, and diagram based methodologies. Highlight space-based grouping methodologies catch the worldwide qualities of the picture through the choice and computation of the picture highlights, which are generally in light of the shading or surface. By utilizing a particular separation measure that disregards the spatial data, the element tests are taken care of as vectors, and the goal is to gathering them into minimized, yet very much isolatedbunches[2].

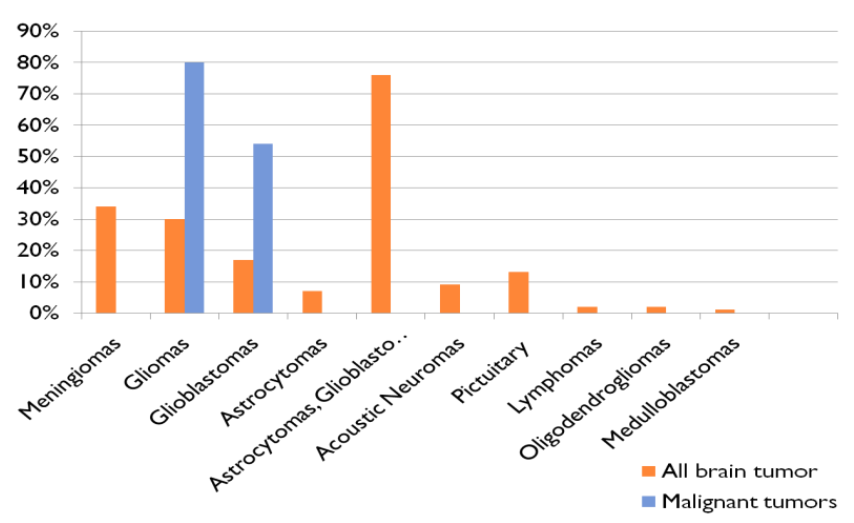

Fig 3: Percentage statistic of different tumors surveyed during year 2015 ${ }^{[25]}$

\section{MRI SCANNER WITH VARIOUS TESLA VALUES}

Many people from countries like Kenya, Nigeria, Tanzania, Ghana, Uganda, Sudan and other African countries travel abroad to India for best hospitals for brain tumor surgery. Many patients from countries in middle east like UAE, Kuwait, Qatar, Oman and many from Afghanistan, Iraq, Iran and other countries come to India for affordable brain tumor surgery. 
The advantage of coming to India for them lies in the fact that the cost of brain tumor or even brain cancer surgery in India is much less as compared to what one would pay in countries like UK, USA for such brain cancer surgery. Total population (2013) 1,252,140,000, Probability of dying between 15 and 60 years $\mathrm{m} / \mathrm{f}$ (per 1000 population, 2012) 242/160. Nearly 70,000 new cases of primary brain tumors will be diagnosed this year. More than 4,600 children between the ages of 0-19 will be diagnosed with a brain tumor this year. There are nearly 7,00,000 people living with a brain tumor. This year, nearly 14,000 people will lose their battle with a brain tumor. There are more than 120 types of brain tumors.

Moreover MRI requires a magnetic field that is both strong and uniform. The field strength of the magnet is measured in TESLA(T). The majority of systems operate at 1.5T, commercial systems are available between $0.2 \mathrm{~T}-7 \mathrm{~T}$. The excited hydrogen atoms of body water emit a radio frequency signal which is measured by a receiving coil of the MRI Scanner. As these coils are rapidly switched on and off they create the characteristic repetitive noise of an MRI scan. Increased heat and increased noise with 3.0 T MRI may also be of concern. Compared with a 1.5 T MRI, a 3.0 T MRI is a larger and heavier machine, and so a larger room may be required, with additional structural support.

The 3.0 T MRI may require upgraded magnetic shielding and may require new wave guides as well as increased floor loading at the support feet. $1.5 \mathrm{~T}$ can't be easily converted to 3T and thus may not be giving noiseless result of MRI images. The collection of MRI image dataset will be identified from the MRI centre or from the DICOM website.

Primary observation of T1 weighted and T2 weighted MRI images of brain. Study those images and basic algorithm for different segmentation methods. Identifying different parameters required in implementation. The performance of the mean shift algorithm is greatly affected by the size of the parzen window and the terminating criteria.

These two issues have been taken care of here in a purely statistical framework. The efficiency of this newly developed adaptive clustering has been judged for segmentation of any initially oversegmented images. Extensive experiments on several MRI images have confirmed the effectively of this proposed approach in comparison to some widely used state of the art segmentation methods.

The superiority of the proposed method will be experimented on a large set of MRI images and compared with some well-known Denoising and segmentation models. The suggested algorithm will help removing noise in certain segmentation technique and enhances the overall segmentation performance. So the suggested method is anticipating in enhancement of image quality with less time for computation.

To implement an entire system of automated brain tumor detection and classification. We had separated our aim into modules like: Registration and removal of noise, Brain tumor classification and segmentation, Report generation, Steganography, Watermarking. For the same purpose the detail block diagram for the expected system is shown below in figure 5 .

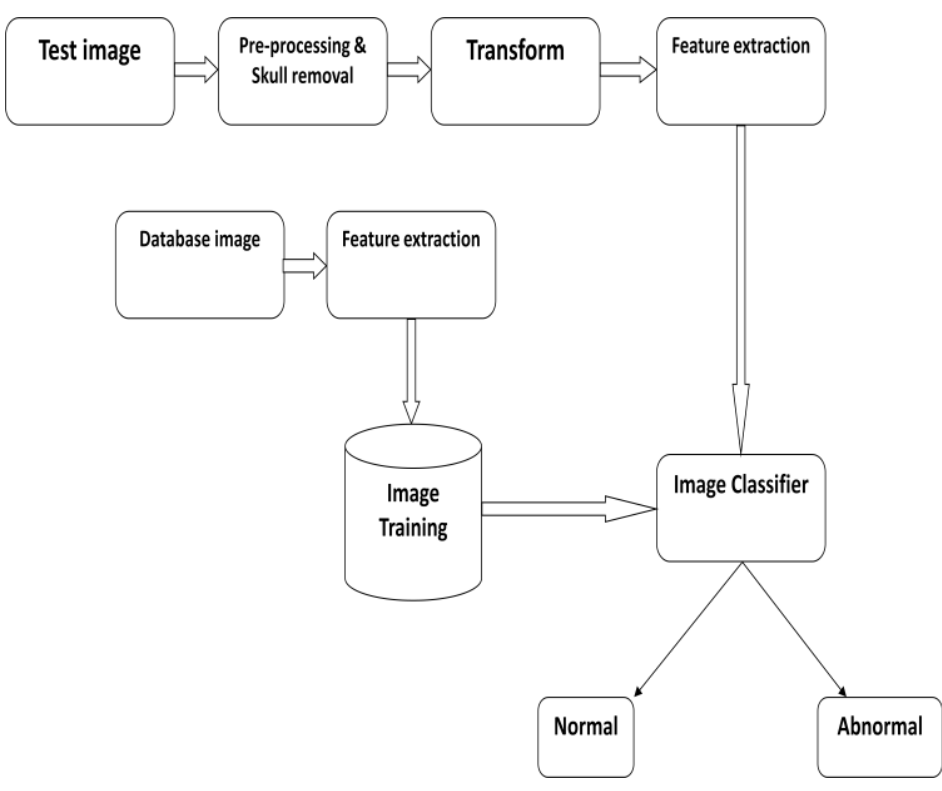

Fig 4: Proposed block diagram for the new system

Test image means input image in this block we had collected scanned image from user to check whether he's a normal or abnormal. Pre-processing \& skull removal will apply filter to remove the noises present in scanned image also remove the skull part from the scanned image for better classification and segmentation of tumor. Then the Transform block will apply any transform to convert our test image in to frequency domain, because in frequency domain only we can extract more texture components, by applying this transform we can archive more accuracy. Feature extraction

Extract the features in transformed image like (texture feature extraction, statistical features) and make a feature vector. The Database image collects the ' $N$ ' no of images from hospital to train our neural network. If we increase the no of database then $\mathrm{u}$ ill archive better accuracy, similar as test image here also we need to apply pre-processing, skull removal and transform. Database feature extraction extracts features from all database images and creates a database feature vector. $\mathrm{NN}$ (Neural network) training is a supervised technique, so output of neural network is already defined by user, similarly one needs to create a target vector and train the database feature vector with target vector using NN.

$\mathrm{NN}$ classifier collects the test image feature vector from user and collect the trained neural network output and simulate both features, finally one can get the result as normal (negative) or abnormal (positive) brain tumor image. Further one can hide the information of the patient whensoever it is needed for the analysis at remote centre. For that stegnography and watermarking also used to make the system more robust.

Table 1 shows the comparison of the different method for Denoising. This Denoising is helpful to remove noise from the input database image which is used for further processing for analysis. This comparison is done after the deep survey of different techniques. The implementation of the best method has been yet to be done. 
Such system can help getting exact information of the patient brain analysis and Medical filed will be having boon if such a system is successfully implemented. Further research will be carried out by the author sooner.

Especially in India the rural area doesn't have efficient scanner and thus it creates problem in analysis of MRI of brain.

Table 1. Assessment by comparison

\begin{tabular}{|c|c|c|}
\hline Method & Activity Iteration & $\begin{array}{c}\text { Computational } \\
\text { Complexity }\end{array}$ \\
\hline DWT & $\begin{array}{c}\text { Transforming, } \\
\text { Threshoding, }\end{array}$ & Less complex \\
\hline TIDAL & $\begin{array}{c}\text { Use split, prediction and } \\
\text { update filter with cycle } \\
\text { spinning algorithm }\end{array}$ & simple \\
\hline TIDFT & $\begin{array}{c}\text { Frame based method, } \\
\text { Use lifting structure } \\
\text { with Atrous algorithm }\end{array}$ & \\
\hline
\end{tabular}

Wavelet transforms seems to be solution to the above problem which are based on small wavelets with restricted durations. Wavelets that decays the statistics into different frequency component. Translated version wavelet trace where we concern and scaled version wavelets permit us to analyze the signal in different scales. From past decade wavelet or subband based transform has been widely discussed. This type of representation should contain basic elements oriented at variety of directions keeping the properties of multiresolution, localization and isotropy as well

Another comparison after the survey is as below in table 2 .

Table 2. Different parameter comparison

\begin{tabular}{|c|c|c|}
\hline Speed & Results & Cost \\
\hline Moderate & $\begin{array}{c}\text { Reduce artifact, preserve } \\
\text { edges }\end{array}$ & Less \\
\hline Fast & $\begin{array}{c}\text { Very good results, high } \\
\text { edge preserving } \\
\text { capability }\end{array}$ & $\begin{array}{c}\text { Economic, not } \\
\text { require machinery } \\
\text { for Fourier analysis }\end{array}$ \\
\hline Faster & $\begin{array}{c}\text { Provide sparse } \\
\text { approximation, edge } \\
\text { preserve in good manner }\end{array}$ & Lesser \\
\hline
\end{tabular}

For wavelet based image Denoising following steps have to perform: 1. Transforming the original image into wavelet domain to get the wavelet coefficient using discrete wavelet transform. 2. Wavelet coefficient has been processed like thresholding the wavelet coefficients to minimize the noise association in the wavelet domain. 3. After captivating the inverse wavelet transform on the processed coefficients produces the denoised image. This step can be repeated for number of wavelet transform scales each representing different degrees of wavelet coefficient.

\section{CONCLUSION}

Picture Denoising is preprocessing stride in picture handling. Like in MRI focus furthermore Astronomical application, pictures are taken and exchanged to diverse areas for examination yet amid obtaining or transmission pictures are adulterated by some clamor basically it is Gaussian added substance irregular commotion. So It is the essential interest for quick calculation and quality concealment of commotion with basic structure. Diverse methodologies for commotion concealment in pictures and sign preparing have been considered. Brief time Fourier change, ceaseless wavelet change and discrete wavelet change have been contemplated. From the hypothesis of wavelet, wavelet coefficient acquires the practices that are topped, since a long time ago tailed dissemination so it is conceivable to model the silent wavelet coefficient. DWT is less mind boggling with intense picture Denoising and have the capacity of edge protecting. Picture Denoising utilizing Discrete Wavelet Transform has been executed. Utilizing this strategy great top sign to commotion has been accomplished. However, DWT is not interpretation invariant which is vital property for picture Denoising. Picture Denoising Using Framelet Transform is executed and enhances PSNR esteem and recuperates the undermined picture with high visual quality. For the future scope the system will be more improved if the automation for removing tumour is successfully done using machine learning.

\section{REFERENCES}

[1] Comaniciu, D.; Meer, P., "Mean shift: a robust approach toward feature space analysis," Pattern Analysis and Machine Intelligence, IEEE Transactions on pattern analysis and machine intelligence, vol.24, no.5, pp.603619, May 2002.

[2] Wenbing Tao, Hai Jin, Yimin Zhang, "Colour Image Segmentation Based on Mean Shift and Normalized Cuts", IEEE Transactions on systems, man, and cybernetics-part b: cybernetics, vol. 37, no. 5, pp.13821389, October, 2007.

[3] Raffaele Gaetano, Giuseppe Scarpa, And Giovanni Poggi, "Hierarchical Texture-Based Segmentation Of Multiresolution Remote-Sensing Images." IEEE Transactions on geoscience and remote sensing, vol. 47, no. 7, pp. 2129-2141, July 2009

[4] Cedric Wemmert, Anne Puissant, Germain Forestier, and Pierre Gancarski, "Multiresolution Remote Sensing Image Clustering," IEEE Geoscience And Remote Sensing Letters, vol. 6, no. 3,pp. 533-537, July 2009.

[5] Pilar Jarabo-Amores, Manuel Rosa-Zurera, David de la Mata-Moya, Raul Vicen-Bueno, and Saturnino Maldonado-Bascon, "Spatial-Range Mean-Shift Filtering and Segmentation Applied to SAR Images.", IEEE Transactions On Instrumentation And Measurement, vol. 60, no. 2,pp. 584-597, February 2011.

[6] Yu-Hsiang Wang, "Tutorial: Image Segmentation", Graduate Institute of Communication Engineering National Taiwan University, Taipei, Taiwan, ROC, Website: www.mathworks.com

[7] Ajala Funmilola A, Oke O.A, Adedeji T.O, Alade O.M, Adewusi E.A, " Fuzzy k-c-means Clustering Algorithm for Medical Image Segmentation", Journal of Information Engineering and Applications, ISSN 22245782 (print) ISSN 2225-0506 (online)Vol 2, No.6, 2012.

[8] Thu Huong Nguyen Fakultät Informatik -TU Dresden, "MEAN SHIFT SEGMENTATION", Proseminar "Aufgabenstellungen der Bildanalyse und Mustererkennung,

[9] Ms. Salve Amrapali Kishanrao,Mr. Salve Avinash P.,Mr Salve Vilas P., "Color Image Segmentation using MSNC Algorithm", International Journal of Engineering Research \& Technology (IJERT) ,ISSN: 2278-0181, Vol. 2 Issue 9, September - 2013. 
[10] Md. Zain, Aryati Binti Bakri,Mahadi Bin Bahari, Pm Dr. Naomie Binti Salim, "Feasibility Study Of Fuzzy Clustering Techniques In Chemical Database For Compound Classification", Universiti Teknologi Malaysia,2006.

[11] Chapter $11 \quad$ Non-Parametric Techniques. http://www.byclb.com/TR/Tutorials/neural_networks/ch 11_1.htm

[12] Jiawei Han,Vipin Kumar, "Clustering",CIS 601 Fall 2004Longin Jan Latecki., Website: http://wwwusers.cs.umn.edu/ kumar/csci5980/index.html

[13] Benjamin James Bush, “ Fuzzy Clustering Techniques: Fuzzy C-Means and Fuzzy Min-Max Clustering Neural Networks", SSIE 617 Term Paper, Fall 2012.

[14] Dr. Mike Spann, "Image Segmentation”,EE4H, M.Sc $0407191 \quad$ Computer Vision, Website:http://www.eee.bham.ac.uk/spannm

[15] Zhaozheng Yin, "Mean shift and feature selection", Spring 2005

[16] Bohyung Han, "Mean-Shift Algorithm and Its Application", Website: bhhan@cs.umd.edu

[17] Leow Wee Kheng, "Mean Shift Tracking", National University of Singapore.

[18] Comaniciu and Meer, "Mean Shift analysis and applications", Proc. ICCV 1999.

[19] Texas A\&M University Website: http://www.research.cs.tamu.edu

[20] S. Sulochana , R.Vidhya , “ Image Denoising using Adaptive Thresholding in Framelet Transform Domain
",(IJACSA) International Journal of Advanced Computer Science and Applications, Vol. 3, 2012

[21] J. Mohan, V. Krishnaveni, Yanhui Guo, "A survey on the magnetic resonance image denoising methods", Biomedical Signal Processing and Control, Vol. 9, pp. 56-69, Elsevier, 2014

[22] Sachin D Ruikar, Dharmpal D Doye, "Wavelet Based Image Denoising Technique", (IJACSA) International Journal of Advanced Computer Science and Applications, Vol. 2, pp. 49-53,March 2011

[23] Yan Shi, Xiaoyuan Yang, and Yuhua Guo, "Translation Invariant Directional Framelet Transform Combined With Gabor Filters for Image Denoising", IEEE Transactions On Image Processing, Vol. 23, pp. 44-55, January 2014

[24] India Times Website: http://timesofindia.indiatimes.com/ home/science/Radiation-may-cause-brain-tumours-laterin-life/articleshow/45043087.cms

[25] About Brain Tumors: A Primer for Patients and Caregivers. http://www.abta.org/secure/about-braintumors-a-primer.pdf

[26] AatmaJyoti MRI Centre, Surat Manav Seva Sangh, "Chhanyado" Sanchalit, New civil Hospital, Majuragate, Surat, Gujarat, India.

[27] Image database: http://www.hopkinsmedicine.org/ healthlibrary/GetImage. aspx?ImageId=161365. gif

[28] Image database: http://www.stanfordchildrens.org/ contentpublic/topic/images/07/161407.gif 